\title{
Relationship between Tibial Bone Defect and Extent of Medial Release in Total Knee Arthroplasty
}

\author{
Shin Woo Nam, MD, Ji Hoon Kwak, MD, Nam Ki Kim, MD, Il Whan Wang, MD and Beom Koo Lee, MD \\ Department of Orthopedic Surgery, Gil Medical Center, Gachon University, Incheon, Korea
}

Purpose: To understand the relationship between tibial bone defect and extent of medial release in total knee arthroplasty.

Materials and Methods: We evaluated 32 knees in 22 patients with variable degrees of tibial bone defect on medial plateau has undergone total knee arthroplasty. In this study, 31 cases had been diagnosed as degenerative osteoarthritis and 1 case was osteonecrosis. We excluded cases with infection, traumatic arthritis, or neuropathic joints. With regard to gender, 29 cases were female and 3 cases were male. The following relationships were analyzed: preoperative degrees of tibial bone defect and varus deformity; the femorotibial angle of both weight-bearing whole extremity radiogram, distractive stress radiogram, and the extent of medial release.

Results: Average tibial bone defect was $9.8 \pm 4.1 \mathrm{~mm}$. Average femorotibial angle on weight-bearing whole extremity radiograph was varus $10.0^{\circ} \pm 6.2^{\circ}$. Average femorotibial angle on distractive stress radiograph was varus $0.7^{\circ} \pm 4.6^{\circ}$. Statistically the extent of medial release showed no significant relationship with the degree of tibial bone defect and femoro-tibial angle of whole extremity radiogram. However, it revealed a statistically significant relationship with the femorotibial angle on distractive radiogram $(r=0.465, \mathrm{p}=0.007)$.

Conclusions: Preoperative distractive stress radiograph might be a useful method to predict the extent of intraoperative medial release during total knee arthroplasty.

Key words: Total knee arthroplasty, Tibial bone defect, Medial release.

\section{Introduction}

Bone defects in knees with advanced osteoarthritis can cause profound varus deformity ${ }^{1)}$, which can lead to medial soft tissue contracture. Depending on the extent of deformity, a medial release is necessary during total knee arthroplasty (TKA) ${ }^{2,3)}$. However, the extent and sequence of medial releases have not been clearly established ${ }^{4-6)}$ and ligament balancing can be

Received June 9, 2011; Revised (1st) July 5, 2011;

(2nd) October 23, 2011; (3rd) December 4, 2011;

Accepted April 12, 2012.

Correspondence to: Beom Koo Lee, MD.

Department of Orthopedic Surgery, Gil Medical Center, Gachon

University, 21 Namdong-daero 774beon-gil, Namdong-gu, Incheon 405760 , Korea.

Tel: +82-32-460-3384, Fax: +82-32-468-5437

Email: bklee@gilhospital.com

This is an Open Access article distributed under the terms of the Creative Commons Attribution Non-Commercial License (http://creativecommons.org/licenses/by-nc/3.0/) which permits unrestricted non-commercial use, distribution, and reproduction in any medium, provided the original work is properly cited. achieved without an extensive medial release even in knees with severe bone defects. The purpose of this study was to evaluate the relationship between the femorotibial angle on the distractive stress radiograph and the extent of medial release in arthritic knees with bone defects.

\section{Materials and Methods}

There were 160 cases that underwent TKA at our institution between January 2006 and January 2009. Of these, 32 cases (22 patients) with a bone defect in the medial proximal tibia were enrolled in this retrospective study. The exclusion criteria were neuropathic arthropathy that may affect soft tissue, traumatic osteoarthritis, infections, valgus deformity, rheumatoid arthritis, past history of surgery, or severe flexion contracture for obtaining a true anteroposterior (AP) view with distractive stress radiography. The mean age of the patients was 69.7 years (range, 48 to 75 years). There were 2 males ( 3 cases) and 20 females (29 cases). The indications for surgery were degenerative osteoarthritis in 31 cases and osteonecrosis in 1 case.

All operations were performed by the same surgeon using a medial parapatellar approach. Tibial and femoral component 
fixation was carried out with cement. Tibial and distal femoral cuts were made perpendicular to the mechanical axis using a measured resection technique in all cases. Sequential medial ligament release was performed using a distractor taking care to maintain the femoral cut surface parallel to the tibial cut surface. Then, anterior/posterior femoral cutting was performed with the knee in $90^{\circ}$ flexion taking care to keep the femoral cut surface parallel to the tibial cut surface. Continuous passive movement was started from the 1st postoperative day. Partial weight-bearing with crutches or a walker was allowed from the 2 nd postoperative day. Full weight-bearing and active joint movement exercises were initiated 1 week after surgery.

Functional assessments were performed using the Knee Society Knee Score (KSKS) and Knee Society Functional Score (KSFS) preoperatively and postoperatively ${ }^{7)}$.

On the radiographic assessment, the extent of bone defects was measured on the AP and lateral views of the knee before surgery and the femorotibial angle was measured on the weight-bearing whole extremity radiograph.

All distractive stress radiographs were taken by the same resident. The patient was placed in the supine position with the leg in neutral position and the examiner pulled the patient's ankle for maximum tension during the taking. The femorotibial angle on the radiograph was measured for assessment.

The radiologic component position was assessed at the last follow-up on the AP and lateral radiographs of the knee using the American Knee Society Roentgenographic Evaluation and Scoring System ${ }^{8}$.

For the assessment of bone defects, a line perpendicular to the
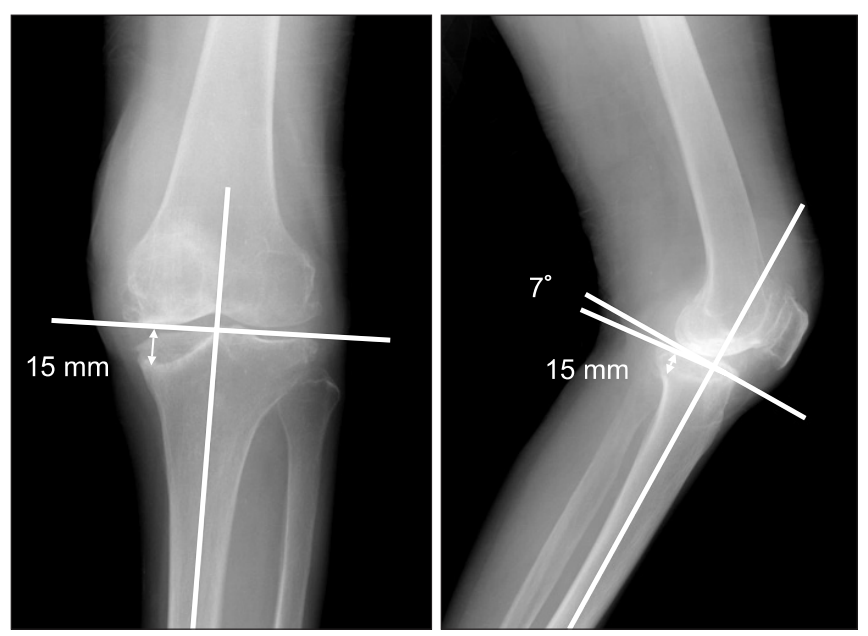

Fig. 1. We measured the tibial medial condylar bone defect in anteroposterior view and lateral view. long axis of the tibia was drawn from a point at the center of the lateral plateau, across the joint surface on the AP view. The perpendicular distance from this line to the deepest portion of the defect in the medial tibial condyle was measured ${ }^{9}$. On the lateral view, considering the posterior slope, a line perpendicular to the long axis of the tibia was drawn across the joint surface from a point in the lateral plateau that creates a posterior slope of $7^{\circ}$. The perpendicular distance from this line to the deepest portion of the defect in the medial tibial condyle was measured. The average value of these 2 measurements was used for evaluation (Fig. 1).

Radiographic measurements were performed twice with an interval of 1 week by 3 observers ( 1 fellow and 2 residents) to assess intraobserver reproducibility and interobserver reliability. The average value of the 6 measurements was used for assessment and statistical analysis.

The extent of medial release was classified into 4 stages and staged medial releases were performed in this study: release of the deep medial collateral ligament (stage 1), release of the posterior oblique ligament or semimembranous insertion (stage 2), release of the posterior capsule (stage 3), and release of the distal superficial collateral ligament or medial epicondylar osteotomy (stage 4$)^{1,10-13)}$.

The improvements in the range of motion, KSKS, and KSFS between the preoperative and postoperative period were assessed using the Wilcoxon rank sum test. The intraobserver reproducibility and interobserver reliability for radiographic measurements were assessed using intraclass correlation coefficients (ICCs). Spearman correlation tests were used to determine the relationships between the extent of a bone defect, varus deformity, and medial release stage, between the femorotibial angle on the preoperative whole extremity radiograph and the medial release stage, and between the femorotibial angle on the distractive stress radiograph and the medial release stage.

\section{Results}

The mean extent of tibial bone defect was $9.8 \pm 4.1 \mathrm{~mm}$. The mean femorotibial angle was $10.0^{\circ} \pm 6.2^{\circ}$ of varus on the whole extremity radiograph and $0.7^{\circ} \pm 4.6^{\circ}$ of varus on the distractive stress radiograph. The mean femorotibial angle at the last followup on the weight-bearing whole extremity radiograph was $7.4^{\circ} \pm 2.5^{\circ}$ of valgus.

The mean ICCs for intraobserver reliability were 0.838 for bone defect measurement, 0.856 for femorotibial angle on the weight- 
Table 1. Intraobserver Reproducibility

\begin{tabular}{lccc}
\hline & $\begin{array}{c}\text { Bone } \\
\text { defect }\end{array}$ & $\begin{array}{c}\text { Femorotibial angle } \\
\text { on standing X-ray }\end{array}$ & $\begin{array}{c}\text { Femorotibial angle } \\
\text { on traction X-ray }\end{array}$ \\
\hline Observer 1 & 0.869 & 0.861 & 0.856 \\
Observer 2 & 0.832 & 0.818 & 0.803 \\
Observer 3 & 0.814 & 0.889 & 0.849 \\
Mean & 0.838 & 0.856 & 0.836 \\
\hline
\end{tabular}

Table 2. Interobserver Reproducibility

\begin{tabular}{lccc}
\hline & $\begin{array}{c}\text { Bone } \\
\text { defect }\end{array}$ & $\begin{array}{c}\text { Femorotibial angle } \\
\text { on standing X-ray }\end{array}$ & $\begin{array}{c}\text { Femorotibial angle } \\
\text { on traction X-ray }\end{array}$ \\
\hline Interobserver $^{\text {a) }}$ & 0.823 & 0.831 & 0.825 \\
Interobserver2 $^{\text {b) }}$ & 0.827 & 0.817 & 0.813 \\
Mean & 0.825 & 0.824 & 0.819
\end{tabular}

Interobserver reliability for the first ${ }^{\mathrm{a})}$ and second ${ }^{\mathrm{b})}$ reading.

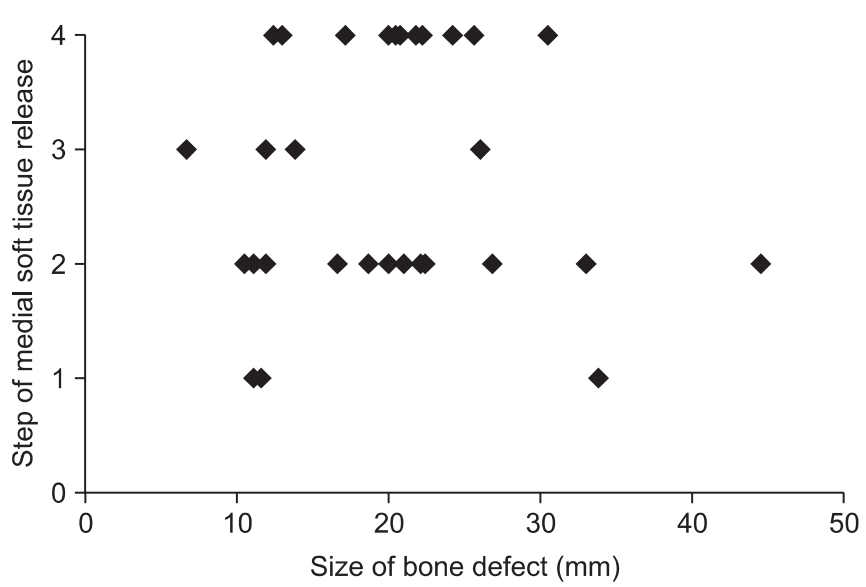

Fig. 2. There is no correlation between the size of bone defect and the degree of medial release.

bearing whole extremity radiograph, and 0.836 for femorotibial angle on the distractive stress radiograph (Table 1).

The mean ICCs for interobserver reliability were 0.825 for bone defect measurement, 0.824 for femorotibial angle on the weightbearing whole extremity radiograph, and 0.819 for femorotibial angle on the distractive stress radiograph (Table 2).

The mean flexion contracture was improved from $5.9^{\circ}$ preoperatively to $0.9^{\circ}$ at the last follow-up ( $\left.<<0.01\right)$. The mean maximal flexion was increased from $106.7^{\circ}$ preoperatively to $121.3^{\circ}$ at the last follow-up ( $\left.\mathrm{p}=0.041\right)$. The mean KSKS was improved from 48.1 preoperatively to 90.4 postoperatively $(\mathrm{p}<0.001)$ and the mean KSFS from 42.9 preoperatively to 86.8 postoperatively $(\mathrm{p}<0.001)$.

No statistically significant relationship was found between the

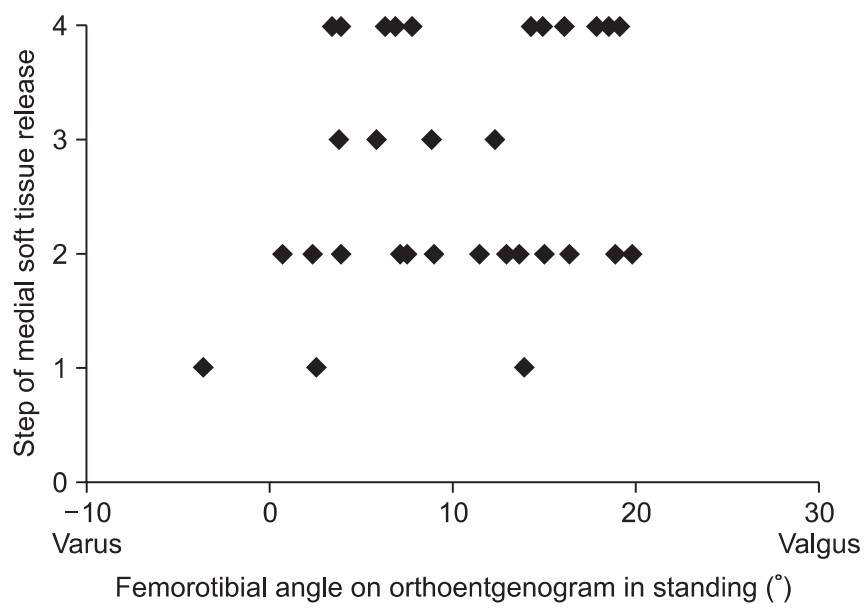

Fig. 3. There is no correlation between femorotibial angle on orthoroentgenogram in standing and step of medial release.

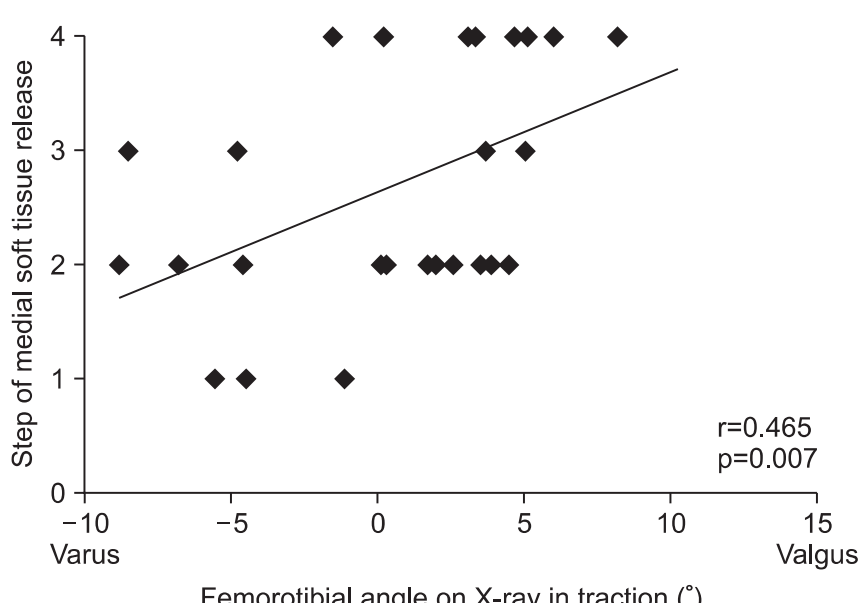

Fig. 4. There is relative correlation between femorotibial angle on orthoroentgenogram in traction and step of medial release.

tibial bone defect and the preoperative femorotibial angle on the weight-bearing whole extremity radiograph (Spearman's correlation coefficient [SCC], $0.210 ; \mathrm{p}=0.248$ ), between the bone defect and the medial release stage (SCC, 0.114; $\mathrm{p}=0.413$ ) (Fig. 2), and between the preoperative femorotibial angle on the weightbearing whole extremity radiograph and the medial release stage (SCC, 0.136; $\mathrm{p}=0.329$ ) (Fig. 3). The femorotibial angle on the distractive stress radiograph was correlated with the medial release stage (SCC, 0.465; p=0.007) (Fig. 4).

\section{Discussion}

The medial aspect of the proximal tibia is a concave area where a bone defect occurs under excessive load with the progression of degenerative arthritis, which eventually results in a varus 
deformity combined with medial soft tissue contracture and lateral soft tissue laxity. Abernethy et al. ${ }^{14)}$ reported that a failure to correct preoperative varus deformity can cause deformation of tibial components due to uneven stress distribution and stress shielding. Mullaji et al. ${ }^{1)}$ mentioned that incomplete correction of preoperative varus deformity can lead to loosening and failure of the tibial component because of the abductor moment on weight bearing that causes gradual collapse of the medial tibial compartment. Therefore, we believe that restoration of proper soft-tissue balance and alignment is essential for successful TKA.

Preoperative assessment of ligament tension is a crucial factor in TKA that helps the surgeon to evaluate the extent of ligament contracture or laxity, determine the stage of medial release, surgical approach, and component type ${ }^{15)}$. Mihalko and Krackow $^{16}$ suggested that preoperative distraction test and varusvalgus stress test can provide useful information on medial and lateral ligament laxity. The difference in joint space width between the lateral and medial compartments on varus-valgus stress radiographs allows the surgeon to assess the medial and lateral ligament laxity before surgery. On the other hand, distraction stress radiographs can be more convenient for the assessment of the laxity because the medial and lateral joint spaces are widened at the same time during the taking of the radiograph. We believe that preoperative radiographic assessment can be effective for preventing medial laxity associated with overcorrection and malalignment associated with undercorrection. The relationship between the extent of release and medial and lateral gap has not been clearly established ${ }^{4-6}$. However, the degree of deformity has been associated with the extent of medial release. Sim et al. ${ }^{10)}$ showed that the extent of medial release was correlated with the degree of varus deformity on preoperative distractive stress radiographs. In our opinion, this is because the more varus the corrected femorotibial angle on the distractive stress radiograph, the more severe the medial ligament contracture that requires an extensive medial release.

Intraoperative assessment of tension is another important element that can be performed using a tension jig, lamina spreaders, or spacer blocks ${ }^{16-18)}$. However, without clear standards for medial release, researchers have suggested a variety of methods for sequential release of medial collateral ligament structures ${ }^{5,6,15)}$. In this study, we performed staged procedures that involve from the deep medial collateral ligament to the superficial collateral ligament release ${ }^{10)}$.

In this study, no statistically significant correlation was found between the extent of preoperative bone defect and degree of varus deformity based on the measurement of the femorotibial angle. In 7 cases with severe bone defects (mean value, 12.7 $\mathrm{mm}$ ) where the mean femorotibial angle on the whole extremity radiograph was $14.6^{\circ}$, we expected extensive medial releases to be performed during TKA. However, more than stage 3 medial releases were not considered necessary during the procedure: stage 1 was carried out in 1 case and stage 2 in 6 cases. In addition, the correlation coefficient was low $(\mathrm{r}=0.114, \mathrm{p}=0.413)$. These results indicate that the extent of medial release is not necessarily related to the extent of bone defects. There was no significant relationship between the varus deformity on the whole extremity radiograph and the extent of medial release. This can be attributable to the flexible components of the medial ligament that allow the medial ligament to be lax when the medial joint space becomes narrow in weight bearing whereas to be taut in flexion when the contact surface is located in the area where bone defects are not present. According to the study by Bae et al. ${ }^{19)}$, the correlation between the varus deformity on the weight-bearing whole extremity radiograph and the probability of measurement error of the mechanical axis can be another reason. However, the corrected varus deformity on the distractive stress radiograph was significantly related to the medial release stage and proper femorotibial angle and ligament balance could be obtained using staged medial releases.

The limitations of this study include that the number of patients was small and the study results cannot be applied to patients with a flexion contracture in whom an AP view of the knee is difficult to obtain with distractive stress radiography. In addition, manual distraction during the taking of the radiography may have compromised the objectivity of the results. However, this could have contributed to creating an environment similar to a real surgical condition where balance is controlled by maximal distraction stress.

\section{Conclusions}

Severe tibial bone defects do not necessarily require extensive medial releases during TKA. Distractive stress radiographs could be more useful than weight-bearing whole extremity radiographs for assessing soft tissue balance to predict the extent of medial release before surgery.

\section{References}

1. Mullaji AB, Padmanabhan V, Jindal G. Total knee arthroplasty for profound varus deformity: technique and radiological results in 173 knees with varus of more than 20 
degrees. J Arthroplasty. 2005;20:550-61.

2. Clayton ML, Thompson TR, Mack RP. Correction of alignment deformities during total knee arthroplasties: staged soft-tissue releases. Clin Orthop Relat Res. 1986;(202):11724.

3. Luring C, Hufner T, Perlick L, Bathis H, Krettek C, Grifka J. The effectiveness of sequential medial soft tissue release on coronal alignment in total knee arthroplasty: using a computer navigation model. J Arthroplasty. 2006;21:428-34.

4. Griffin FM, Insall JN, Scuderi GR. Accuracy of soft tissue balancing in total knee arthroplasty. J Arthroplasty. 2000; 15:970-3.

5. Matsueda M, Gengerke TR, Murphy M, Lew WD, Gustilo RB. Soft tissue release in total knee arthroplasty. Cadaver study using knees without deformities. Clin Orthop Relat Res. 1999;(366):264-73.

6. Yagishita K, Muneta T, Ikeda H. Step-by-step measurements of soft tissue balancing during total knee arthroplasty for patients with varus knees. J Arthroplasty. 2003;18:313-20.

7. Insall JN, Dorr LD, Scott RD, Scott WN. Rationale of the Knee Society clinical rating system. Clin Orthop Relat Res. 1989;(248):13-4.

8. Ewald FC. The Knee Society total knee arthroplasty roentgenographic evaluation and scoring system. Clin Orthop Relat Res. 1989;(248):9-12.

9. Brand MG, Daley RJ, Ewald FC, Scott RD. Tibial tray augmentation with modular metal wedges for tibial bone stock deficiency. Clin Orthop Relat Res. 1989;(248):71-9.

10. Sim JA, Kwak JH, Yang SH, Moon SH, Lee BK, Kim JY. Utility of preoperative distractive stress radiograph for beginners to extent of medial release in total knee arthroplasty. Clin Orthop Surg. 2009;1:110-3.

11. Vail TP, Lang JE. Surgical techniques and instrumentation in total knee arthroplasty. In: Scott WN, ed. Surgery of the knee. 4th ed. New York: Churchill Livingstone; 2006. p1494521.

12. Peters CL. Soft-tissue balancing in primary total knee arthroplasty. Instr Course Lect. 2006;55:413-7.

13. Engh GA. Medial epicondylar osteotomy: a technique used with primary and revision total knee arthroplasty to improve surgical exposure and correct varus deformity. Instr Course Lect. 1999;48:153-6.

14. Abernethy PJ, Robinson CM, Fowler RM. Fracture of the metal tibial tray after Kinematic total knee replacement. A common cause of early aseptic failure. J Bone Joint Surg Br. 1996;78:220-5.

15. Bottros J, Gad B, Krebs V, Barsoum WK. Gap balancing in total knee arthroplasty. J Arthroplasty. 2006;21:11-5.

16. Mihalko WM, Krackow KA. Flexion and extension gap balancing in revision total knee arthroplasty. Clin Orthop Relat Res. 2006;446:121-6.

17. Freeman MA, Todd RC, Bamert P, Day WH. ICLH arthroplasty of the knee: 1968-1977. J Bone Joint Surg Br. 1978;60:339-44.

18. Insall JN, Binazzi R, Soudry M, Mestriner LA. Total knee arthroplasty. Clin Orthop Relat Res. 1985;(192):13-22.

19. Bae DK, Song SJ, Yoon KH, Chung KY. Postoperative limb alignment after total knee arthroplasty depending on the severity of the varus knee. J Korean Knee Soc. 2008;20:13742. 